\title{
A influência dos terceiros molares no apinhamento de incisivos inferiores
}

\author{
Kallil Vinícios Santos TEIXEIRA'; Maria Eleuza Alves SOUZA²; Tatyane \\ Guimarães Ribeiro de CASTRO ${ }^{3}$; Allisson Filipe Lopes MARTINS ${ }^{4}$
}

1 - Acadêmico do curso de Odontologia da Faculdade União de Goyazes, Trindade/Go - Brasil; 2 - Acadêmico do curso de Odontologia da Faculdade União de Goyazes, Trindade/GO - Brasil; 3 - Ortodontista, CORUS Odontologia, Goiânia/GO - Brasil; 4 Professor de Diagnóstico Bucal e Clínica Infantil da Faculdade União de Goyazes, Trindade/GO - Brasil.

\section{Resumo}

Introdução: A época de erupção dos terceiros molares inferiores pode coincidir com o apinhamento dos incisivos inferiores. O terceiro molar como um dos fatores etiológicos do apinhamento anteroinferior ainda não é completamente aceita e pode levar a tratamentos desnecessários e incerteza dos resultados do tratamento ortodôntico. Objetivo: Correlacionar o espaço retromolar e o apinhamento dentário anteroinferior. Material e Métodos: Foram avaliados 21 pacientes com maloclusão de classe I de Angle, que apresentavam radiografia cefalométrica em norma lateral e modelos de estudo de gesso. Foram coletadas informações sobre idade, sexo, etnia. Foram determinados o padrão de crescimento facial e a distância retromolar dos participantes por meio de análise da radiografia cefalométrica, o índice de irregularidade de Little foi calculado, a partir da análise de modelos, para determinar a severidade do apinhamento dentário. A radiografia panorâmica foi utilizada para avaliar a presença e a posição dos terceiros molares. Resultados: O padrão mesofacial foi o mais frequente (66,7\%), seguido pelo braquifacial (33,3\%). A maioria dos pacientes apresentavam apinhamento moderado $(52,4 \%)$, seguido por apinhamento mínimo $(28,6 \%)$. Não houve diferença estatisticamente significante no índice de Little considerando a tipologia facial. Não houve correlação entre o espaço retromolar e o índice de Little. Conclusão: Não há correlação significativa entre o espaço retromolar e apinhamento dentário, sugerindo que a presença do terceiro molar não tem influência no apinhamento anteroinferior.

PALAVRAS-CHAVE: Terceiro Molar; Má oclusão; Ortodontia.

AUTOR PARA CORRESPONDÊNCIA

Allisson Filipe Lopes Martins

Faculdade União de Goyazes, Trindade, Goiás, Brasil.

Telefone: +5562981245239

E-mail: allissonflipe@hotmail.com 


\section{Introdução}

O apinhamento dentário é uma das condições mais frequentes no consultório do ortodontista. Sugere-se que a largura e comprimento dos arcos dentários influenciem no apinhamento, sendo que é mais frequente em mulheres ${ }^{1}$. Além disso, fatores esqueléticos (crescimento maxilar e má oclusão), fatores dentários (tamanho da coroa dentária e perda dentária primária) e fatores gerais (idade e sexo) devem ser avaliados em pacientes com apinhamento dentário, possibilitando diagnóstico mais preciso ${ }^{1,2}$. Estudos recentes têm tentando demonstrar a influência dos terceiros molares no apinhamento anteroinferior e os resultados são controversos ${ }^{2-5}$.

O apinhamento tardio dos incisivos inferiores pode ocorrer simultaneamente à erupção dos terceiros molares inferiores ${ }^{3,6}$. No entanto, a real influência dos terceiros molares inferiores no apinhamento anteroinferior ainda é desconhecida ${ }^{1}$. Esan e Schepartz ${ }^{3}$ (2017) observaram que apenas a presença dos terceiros molares e sua posição quando impactados não são úteis para determinar a ocorrência do apinhamento anteroinferior, sugerem que essa condição é multifatorial.

Acredita-se que a presença dos terceiros molares não é a única etiologia para o apinhamento anterior, pois a diminuição do espaço no arco inferior pode ocorrer mesmo na ausência dos terceiros molares, sugere-se que mesmo quando não há espaço disponível no arco, o terceiro molar pode permanecer impacta$\mathrm{do}^{3}$. No entanto, há autores que sugerem que a inclinação mesial dos terceiros molares pode ocasionar uma força mesial nos dentes do arco inferior, resultando em apinhamento ${ }^{4,5,7}$.

A extração dos terceiros molares com indicação de evitar o apinhamento tardio dos incisivos inferiores também tem sido debatida. Para Karasawa et al. ${ }^{8}$ (2013) não há evidências que incriminam ou que apoiam a remoção profilática de terceiros molares impactados assintomáticos, além disso o real papel do terceiro molar no apinhamento dentário anteroinferior não está 
completamente esclarecido. É importante ressaltar que estudos que investiguem a etiologia da falta de alinhamento dentário na região anterior do arco pode indicar ou evitar procedimentos preventivos, tais como a extração de terceiros molares 9 .

Diante disso, o objetivo deste estudo foi avaliar a influência dos terceiros molares inferiores no apinhamento anteroinferior, com intuito de melhor compreensão da etiologia dessa condição, evitando diagnósticos errôneos, favorecendo terapêuticas apropriadas.

\section{Material e métodos}

Esta investigação trata-se de um estudo transversal com pacientes com apinhamento anteroinferior tratados em uma clínica particular de Goiânia durante o período de 2015 a 2020.

\section{Seleção dos pacientes}

A amostra do presente estudo foi de conveniência, foram selecionados os pacientes do arquivo de uma clínica particular, os quais foram atendidos no período compreendido entre os anos de 2015 a 2020.

Os critérios de inclusão foram: pacientes de ambos os sexos, com idade entre 16 e 29 anos, com má oclusão de classe I de Angle, apinhamento dos incisivos inferiores, com terceiros molares presentes, que tinham disponível radiografia cefalométrica em norma lateral e modelos de estudo de gesso. Os critérios de exclusão aplicados foram: pacientes com distúrbios sistêmicos, presença de próteses dentárias fixas (pônticos, coroas fixas), pacientes com ausências dentárias, pacientes previamente submetidos a tratamento ortodôntico ou ortopédico dos maxilares, pacientes submetidos à extração dos terceiros molares inferiores.

\section{Dados clínico-demográficos e de imagem}

Os dados clínico-demográficos e de imagens foram coletados a partir dos prontuários e imagens radiográficas constantes nos prontuários da clínica em que o estudo foi realizado. Foram coletadas informações sobre idade e sexo. A radiografia cefalométrica em norma lateral foi utilizada para determinar o padrão de crescimento facial e a distância retromolar dos participantes. A 
radiografia panorâmica foi utilizada para avaliar a presença e a posição dos terceiros molares. O modelo de estudo de gesso foi utilizado para calcular o índice de irregularidade de Little.

\section{Traçado cefalométrico}

O traçado cefalométrico, com imagens digitais ou digitalizadas, foi realizado utilizando o software Image $J^{10}$ para auxiliar na determinação do padrão facial e no espaço retromolar. O desenho e a análise cefalométrica foram realizados por um único pesquisador previamente treinado, em ambiente de penumbra e favorável. A padronização das distâncias se deu utilizando a régua disponível nas radiografias.

Primeiramente, foram identificados os pontos cefalométricos: Orbital (Or) - Ponto localizado na parte mais inferior da borda externa da cavidade orbitária; Pório (Po) - Ponto localizado na parte mais superior do meato acústico externo ou conduto auditivo externo; Pterigoídeo (Pt) - Intersecção do bordo inferior do forame redondo com a parede posterior da fissura pterigomaxilar; Gônio (Go) - É o ponto mais posterior e inferior da mandíbula, no sentido anteroposterior. Está localizado na bissetriz do ângulo formado pelas tangentes à borda inferior do corpo e borda posterior do ramo ascendente da mandíbula; Mentoniano (Me) - Ponto mais inferior do contorno externo da sínfise mandíbula; $\mathrm{D}(7)$ - Ponto localizado na parte mais distal da coroa do segundo molar inferior.

Após isso, foram traçados os planos de Frankfurt - linha traçada que une os pontos (Or) e (Po); Plano Mandibular - linha traçada que une os pontos (Go) e (Me) e; Plano oclusal - linha traçada sobre a intersecção entre os dentes superiores e inferiores.

\section{Classificação do padrão facial}

Os participantes da pesquisa foram classificados de acordo com o padrão de crescimento facial em dólicofacial, mesofacial ou braquifacial. Para tanto, foi utilizada a informação constante na 
documentação ortodôntica, relativa ao cálculo do VERT, disponível na análise de Ricketts. O ângulo formado pela intersecção do plano mandibular com o plano horizontal (ângulo FMA) foi utilizado para esta classificação, quando o cálculo do VERT não estava disponível.

\section{Espaço retromolar}

Para o cálculo do espaço retromolar, foi construído na cefalometria o ponto Xi. Este ponto é localizado no centro geográfico do ramo ascendente da mandíbula e é considerada a referência póstero-inferior dos eixos internos da mandíbula ${ }^{11}$. A localização do "Xi" foi realizada obedecendo aos seguintes passos:

1) Determinou-se os pontos R1, R2, R3, R4 na mandíbula:

R1 - Ponto mais profundo da curvatura da borda anterior do ramo ascendente;

R2 - Ponto localizado no bordo posterior da mandíbula, oposto a R1 e paralelo ao plano de Frankfurt;

R3 - Ponto no centro e na parte mais reentrante da chanfradura sigmoide do ramo;

R4 - Ponto no bordo inferior da mandíbula, oposto a R3 e paralela a vertical pterigoidea.

2) Em seguida, foi traçado duas retas paralelas a Frankfurt e que passasse por R1 e R2.

3) Após isso, duas retas paralelas a vertical pterigoidea e que passasse por R3 e R4 foram traçadas.

4) Formou-se um retângulo que engloba o ramo mandibular.

5) O ponto "Xi" é localizado no centro deste retângulo pela intersecção de suas diagonais ${ }^{11}$.

A figura 1 mostra os pontos, planos e medidas cefalométricas que foram utilizadas. 


\section{Posição dos terceiros molares}

Os terceiros molares presentes foram classificados, de acordo com a classificação de Winter ${ }^{12}$, em: vertical, mesioangular, distoangular, horizontal, vestibulo-angular, linguo-angular e invertido.

Índice de irregularidade de little

O índice de irregularidade de Little, o qual reflete o nível de apinhamento do paciente, foi calculado pela soma das distâncias lineares entre os pontos de contato anatômico dos dentes anteriores da mandíbula, utilizando fotos dos modelos inferiores, no software ImageJ. Para padronização das distâncias, uma marcação em cima das bordas incisais dos incisivos com exatos $1 \mathrm{~cm}$ foi realizada. Após isso, foi realizada a soma das distâncias obtidas, o resultado obtido foi útil para classificar o apinhamento/irregularidade como: 0 - alinhamento perfeito; 1 - 3: irregularidade mínima; 4 - 6: irregularidade moderada; 7 - 9: irregularidade severa; 10: irregularidade muito severa ${ }^{13}$. A Figura 2 demonstra o estudo do modelo de gesso no software ImageJ.

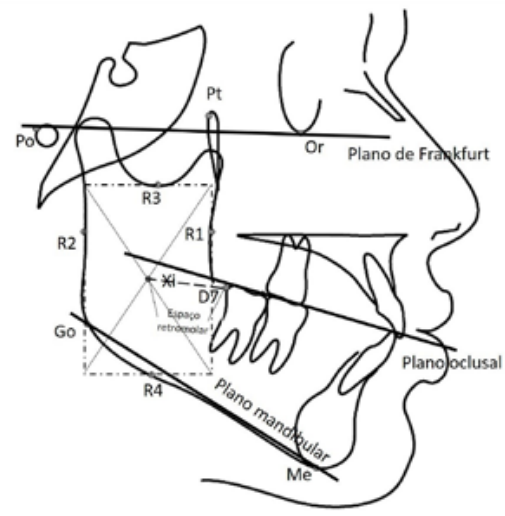

FIGURA 1 - Medidas cefalométricas do estudo. Abreviações: Po - ponto pório; Pt - ponto pterigoide; Or - ponto orbital; R3 - ponto R3; R2 - ponto R2; Xi - ponto Xi; D7 - superfície mais distal da coroa do segundo molar; R1 - ponto R1; Go ponto gônio; R4 - ponto R4; Me - ponto mentoniano.

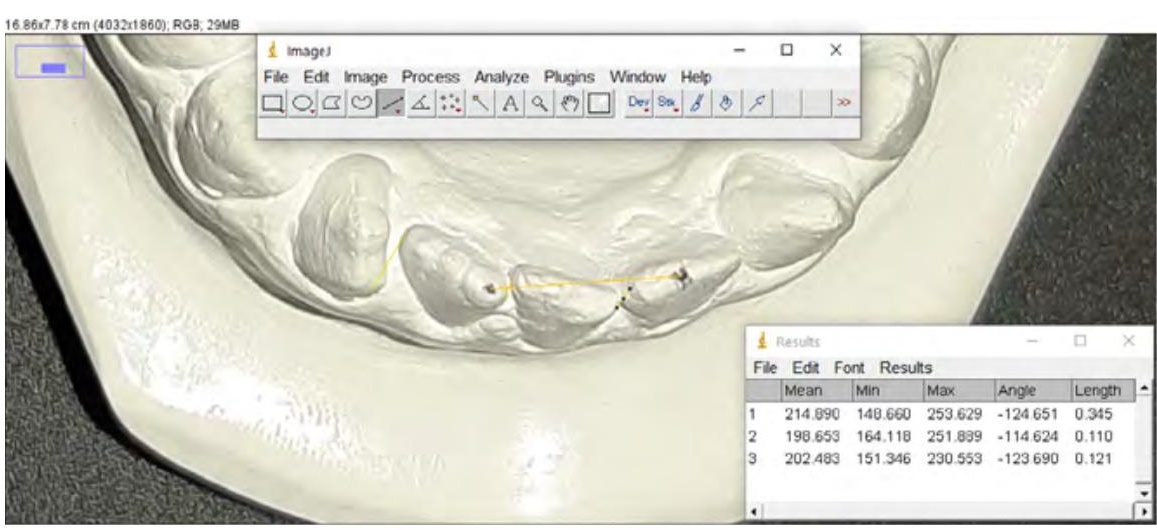

FIGURA 2 - Análise do índice de Irregularidade de Little no software ImageJ. As linhas amarelas indicam as distâncias lineares entre os pontos de contato anatômico dos dentes anteriores da mandíbula. Os pontos desenhados a lápis no modelo correspondem a um centímetro e foram utilizados para padronização das distâncias. 


\section{Análise estatística}

A comparação das características clínicas e demográficas dos pacientes foi realizada por meio do teste de Pearson Quiquadrado para as variáveis categóricas. Para a comparação das variáveis numéricas, foi realizado o teste t de Student, devido à distribuição normal das variáveis. O índice de irregularidade de Little foi comparado com o teste do Qui-Quadrado e teste t de Student. A correlação entre o índice de irregularidade de Little e o espaço retromolar foi calculada a partir do teste de correlação de Pearson. Os resultados estão apresentados como média \pm desvio padrão e frequências absolutas e relativas. Foi utilizado o software estatístico IBM SPSS 20.0 (Nova York, EUA), sendo que valores de $p$ menores que 0,05 foram considerados estatisticamente significantes.

\section{Resultados}

Foram incluídos neste estudo 21 participantes, 12 do sexo feminino $(57,1 \%)$ e 9 do masculino $(42,9 \%)$. A maioria dos participantes apresentavam padrão mesofacial (14/66,7\%), o padrão braquifacial foi observado em 7 participantes (33,3\%), nenhum paciente incluído no estudo era dolicofacial. A média ( \pm d.p.) de idade dos participantes foi de $16,38 \pm 3,18$. A média de idade dos pacientes do sexo feminino foi de $16,33 \pm 3,60$ e do sexo masculino foi de 16,42 $\pm 2,99$ ( $\mathrm{p}=0,47$; Teste $\mathrm{t}$ de Student).

Os terceiros molares apresentavam-se impactados na maioria dos casos ( $\mathrm{n}=19 / 90,5 \%)$, somente dois pacientes apresentavam os terceiros molares erupcionados ( $n=2 / 9,5 \%$ ). De acordo com a classificação de Winter ${ }^{13}$, os terceiros molares apresentavam-se verticalizados em 6 pacientes (28,6\%), mesio-angulados em $13(61,9 \%)$ e vestíbulo-angulados em 2 (9,5\%).

O cálculo do Índice de Little demonstrou que a maiorias dos pacientes apresentavam um índice de irregularidade moderado $(11 / 52,4 \%)$. Seis pacientes apresentaram irregularidade mínima 
$(28,6 \%)$, a irregularidade severa e muito severa foram observadas em $3(14,3 \%)$ e $1(4,8 \%)$ pacientes, respectivamente.

Associação entre perfil facial e espaço retromolar Considerando o perfil facial, a média do espaço retromolar, calculado como a distância do ponto Xi até a o ponto mais distal do segundo molar, foi de $31,06 \pm 9,53$ em pacientes braquifaciais e 27,51 \pm 9,69 em pacientes mesofaciais. Não foram observadas diferenças estatisticamente significantes do espaço retromolar entre pacientes braquifaciais e mesofaciais $(\mathrm{p}=0,37$; Teste $\mathrm{t}$ de Student). A tabela 1 resume os dados sobre espaço retromolar e índice de Little de acordo com o perfil facial do paciente.

Associação entre a posição do terceiro molar e espaço retromolar

O espaço retromolar de pacientes com os terceiros molares mesio-angulados foi maior do que pacientes com esses dentes em posição vertical ou vestíbulo-angulada, no entanto essa diferença não foi estatisticamente significante (Tabela 1).

TABELA 1 • Espaço retromolar e índice de Little dos pacientes avaliados

\begin{tabular}{|c|c|c|c|c|c|}
\hline & & Espaço retromolar & $p^{\star}$ & Índice de Little & $p^{\star}$ \\
\hline \multirow{2}{*}{$\begin{array}{l}\text { Tipologia } \\
\text { facial }\end{array}$} & Braquifacial $(n=7)$ & $31,06 \pm 9,53$ & \multirow{2}{*}{0,37} & $4,47 \pm 2,80$ & \multirow{2}{*}{0,33} \\
\hline & Mesofacial $(n=14)$ & $27,51 \pm 9,69$ & & $5,67 \pm 2,53$ & \\
\hline \multirow{2}{*}{ Sexo } & Feminino $(n=12)$ & $27,35 \pm 9,29$ & \multirow{2}{*}{0,76} & $5,57 \pm 2,98$ & \multirow{2}{*}{0,23} \\
\hline & Masculino $(n=9)$ & $30,90 \pm 10,19$ & & $4,87 \pm 2,15$ & \\
\hline \multirow{3}{*}{$\begin{array}{l}\text { Posição } \\
\text { do terceiro } \\
\text { molar }\end{array}$} & Vertical $(n=6)$ & $25,37 \pm 8,60$ & \multirow{3}{*}{0,28} & $4,37 \pm 1,75$ & \multirow{3}{*}{$0,26^{\star \star}$} \\
\hline & Mesio-angulado $(n=13)$ & $30,41 \pm 9,58$ & & $5,76 \pm 2,68$ & \\
\hline & Vestíbulo-angulado $(n=2)$ & $29,39 \pm 16,35$ & & $4,81 \pm 5,21$ & \\
\hline
\end{tabular}

* Teste T de Student.

**0s molares vestíbulo-angulados não foram incluídos na comparação estatística devido ao número limitado. 
Associação entre o índice de little e a tipologia facial

Os pacientes mesofaiciais apresentaram um índice de Little de $5,67 \mathrm{~mm}$ enquanto que para os braquifaciais, este índice foi de 4,47 , no entanto não foram encontradas diferenças estatisticamente significantes quando comparados os perfis faciais (Tabela 1).

Considerando a classificação do índice de Little como mínima, moderada ou severa, verificou-se que a irregularidade moderada foi mais frequente nos pacientes mesofaciais, enquanto que nos pacientes braquifaciais, irregularidade mínima e moderada apresentaram a mesma distribuição (Tabela 2). Não houve diferença estatisticamente significante do índice de Little considerando a tipologia facial ( $\mathrm{p}=0,69$; teste do Qui-quadrado).

Associação entre o índice de little e a posição do terceiro molar Pacientes com terceiros molares mesio-angulados apresentaram os maiores valores de índice de irregularidade de Little, seguidos por pacientes com esses dentes vestíbulo-angulados. Pacientes com os terceiros molares em posição vertical apresentaram menor média do índice de Little, no entanto, não foram encontradas diferenças estatisticamente significativas entre os grupos (Tabela 1). Não foram observadas associações entre os escores do Índice de Little e a posição do terceiro molar de acordo com classificação de Winter (Tabela 3 ).

TABELA 2 - Escores do Índice de Little de acordo com a tipologia facia

\begin{tabular}{|c|c|c|c|}
\hline & $\begin{array}{l}\text { Braquifacial } \\
(n=7)\end{array}$ & $\begin{array}{l}\text { Mesofacial } \\
(n=14)\end{array}$ & Valor de $p^{*}$ \\
\hline Irregularidade mínima & $3(42,9 \%)$ & $3(21,4 \%)$ & \multirow{4}{*}{0,69} \\
\hline Irregularidade moderada & $3(42,9 \%)$ & $8(57,1 \%)$ & \\
\hline Irregularidade severa & $1(14,3 \%)$ & $2(14,3 \%)$ & \\
\hline Irregularidade muito severa & 0 & $1(7,1 \%)$ & \\
\hline
\end{tabular}

* Teste do Qui-quadrado. 
Associação entre índice de little e sexo

Considerando o sexo, a média do índice de Little foi de 5,57 \pm 2,98 em pacientes do sexo feminino e 4,87 $\pm 2,14$ em pacientes do sexo masculino ( $\mathrm{p}=0,23$; Teste $\mathrm{t}$ de Student). A figura 3 ilustra a distribuição dos escores do Índice de Irregularidade de Little de acordo com o sexo do paciente.

Correlação entre índice de little e espaço retromolar

Uma correlação negativa e fraca $(r=-0,27)$ foi observada entre o índice de Little e o espaço retromolar, no entanto essa correlação não alcançou significância estatística $(\mathrm{p}=0,23$; Teste de Correlação de Pearson) (Figura 4).

\section{Discussão}

No presente estudo foi investigada a associação entre a posição e o espaço retromolar e o apinhamento anteroinferior, nossos resultados sugerem que não, que a posição dos terceiros molares inferiores, o espaço retromolar e a tipologia facial não estão associadas com o apinhamento dos incisivos inferiores em pacientes jovens. Sugere-se que outros fatores podem estar associados com esta má oclusão, tais como o estreitamento da dimensão intercanina, redução do comprimento do arco dental, formato e tamanho dos dentes, contatos dentais e as alterações mandibulares que ocorrem na adolescência ${ }^{2}$.

TABELA 3 - Escores do Índice de Little de acordo com a posição do terceiro molar

\begin{tabular}{|c|c|c|c|}
\hline & $\begin{array}{l}\text { Vertical } \\
(n=6)\end{array}$ & $\begin{array}{l}\text { Mesio-angulado } \\
(n=13)\end{array}$ & Valor de p* \\
\hline Irregularidade mínima & $2(33,3 \%)$ & $3(23,1 \%)$ & \multirow{4}{*}{0,53} \\
\hline Irregularidade moderada & $4(66,7 \%)$ & $7(53,8 \%)$ & \\
\hline Irregularidade severa & 0 & $2(15,4 \%)$ & \\
\hline Irregularidade muito severa & 0 & $1(7,7 \%)$ & \\
\hline
\end{tabular}

* Teste do Qui-quadrado 
Nossos resultados corroboram com Karasawa et al. ${ }^{8}$ (2013) e Hasegawa et al. ${ }^{14}$ (2012), os quais também não verificaram relação entre terceiros molares e apinhamento dos incisivos inferiores. No entanto, Selmani et al. ${ }^{4}$ (2016), sugerem que há uma forte relação entre a angulação e posição do terceiro molar com o apinhamento do arco inferior. Vale ressaltar que os autores levaram em consideração o índice de Ganss, o qual calcula a distância retromolar em radiografias panorâmicas. Apesar do índice de Ganss servalidado ${ }^{15}$ e utilizado para calcular o espaço retromolar ${ }^{4,14}$, acreditamos que as distorções inerentes à radiografia panorâmica podem levar a resultados equivocados, nesse sentido calculamos o espaço retromolar utilizando a radiografia cefalométrica em norma lateral, a qual é amplamente utilizada em estudos para calcular medidas lineares e angulares ${ }^{16-18}$.

Zawawi e Melis ${ }^{6}$ (2014) sugerem que a erupção dos terceiros molares inferiores e o apinhamento anteroinferior ocorrem simultaneamente, no entanto são dois eventos diferentes. Além disso, sugere-se que as alterações nos arcos dentários podem ser responsáveis pelo apinhamento anteroinferior ${ }^{19}$. Adicionalmente, Tsiopas et al. ${ }^{20}$ (2013) acompanharam dezoito adultos e observaram alterações dento-alveolares e oclusais ocorridas ao longo de 38,4 anos de acompanhamento dos pacientes.

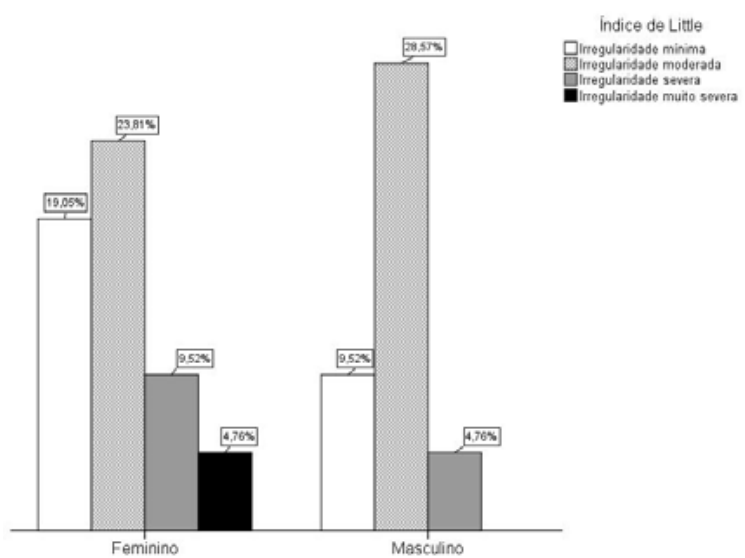

FIGURA 3 - Índice de irregularidade de Little de acordo com o sexo dos participantes.

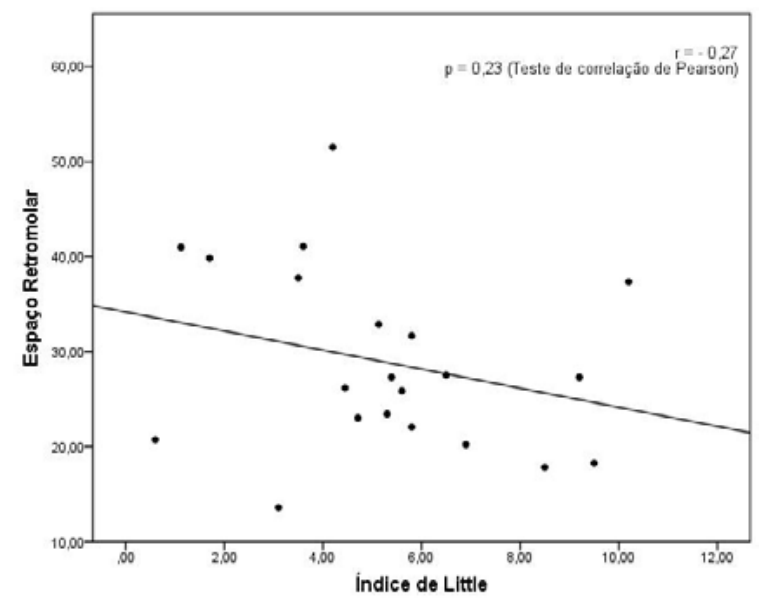

FIGURA 4 • Diagrama de dispersão do Espaço Retromolar versus Índice de Little. 
As principais alterações encontradas foram diminuições no comprimento e profundidade do arco, resultando em uma diminuição da largura intercanina e um aumento no apinhamento anterior, sugerindo que as alterações dento-alveolares ocorrem como um processo contínuo ao longo da vida adulta, ressalta-se ainda que essas alterações possam levar ao apinhamento anteroinferior.

Nossos achados demonstraram ainda que não há associações entre a posição do terceiro molar e o apinhamento anteroinferior. Nossos dados são semelhantes aos encontrados por Chaves-Neto et al. ${ }^{19}$ (2010), e reforçam que não há justificativa para indicação de remoção dos terceiros molares, mesmo que em posições desfavoráveis, com a finalidade de prevenir o apinhamento anteroinferior.

A extração dos terceiros molares para prevenir o apinhamento dentário tem sido estudada. Lindauer et al. ${ }^{21}$ (2007) verificaram que profissionais formados entre os anos de 1970 e 1980 são mais propensos a acreditar que os terceiros molares influenciam no apinhamento, deste modo, a recomendação de extração profilática dos terceiros molares ${ }^{21}$. De modo contrário, Tüfekçi et al. ${ }^{22}$ (2009) e Gavazzi et al. ${ }^{23}$ (2014) verificaram que os profissionais acreditam que não há justificada para remoção profilática dos terceiros molares para evitar o apinhamento anteroinferior. Esses estudos não foram realizados com cirurgiões-dentistas brasileiros, sugerimos que sejam realizados estudos que investiguem a opinião do profissional sobre a indicação de exodontia dos terceiros molares para prevenção de apinhamento anteroinferior.

Devido aos critérios de inclusão adotados neste estudo, para evitar vieses de seleção da amostra, o número amostral limitado pode ser visto como uma limitação do estudo. Acreditamos que estudos futuros com a inclusão de um maior número de pacientes, incluindo dólicofaciais, possa fortalecer nossos achados sobre a real influência dos terceiros molares inferiores no apinhamento anteroinferior. 


\section{Conclusão}

No presente estudo, demonstramos que o apinhamento do anteroinferior não está associado com a posição ou com espaço disponível para erupção dos terceiros molares inferiores. No entanto, pesquisas futuras, incluindo um maior número de participantes e pacientes dólicofaciais devem ser realizadas.

\section{Referências}

1 - Stanaitytė R, Trakinienė G, Gervickas A. Do wisdom teeth induce lower anterior teeth crowding? A systematic literature review. Stomatologija. 2014; 16(1): 15-18.

2- Sidlauskas A, Trakiniene G. Effect of the lower third molars on the lower dental arch crowding. Stomatologija. 2006; 8(3): 80-84.

3 - Esan T, Schepartz LA. Third molar impaction and agenesis: influence on anterior crowding. Ann Hum Biol. 2017; 44(1): 46-52.

4 - Selmani ME, Gjorgova J, Selmani ME, Shkreta M, Duci SB. Effects of Lower Third Molar Angulation and Position on Lower Arch Crowding. Int J Orthod. 2016; 27(1): 45-49.

5 - Niedzielska I. Third molar influence on dental arch crowding. Eur J Orthod. 2005; 27(5): 518-523.

6 - Zawawi HK, Melis M. The Role of Mandibular Third Molars on Lower Anterior Teeth Crowding and Relapse after Orthodontic Treatment: A Systematic Review. ScientificWorldJournal, 2014; 2014:615429

7 - Zachrisson BU. Mandibular third molars and late lower arch crowding-the evidence base. World J Orthod. 2005; 6(2):180-186.

8 - Karasawa LH, Rossi AC, Groppo FC, Prado FB, Caria PHF. Crosssectional study of correlation between mandibular incisor crowding and third molars in young Brazilians. Med Oral Patol Oral Cir Bucal. 2013; 18(3):e505-e509.

9 - Mattos RMPR, Sotero SF, Franco AA, Carvalho RWF, Falcão PGCB. A influência do terceiro molar no apinhamento antero-inferior. Rev Cir Traumatol Buco-Maxilo-Fac. 2008; 8(3):9-16.

10 - Schneider, C. A.; Rasband, W. S. Eliceiri, K. W. NIH Image to ImageJ: 25 years of image analysis. Nat Methods. 2012; 9(7):671-675.

11 - Borbolla RR, Faltin-Junior K, Costa C, Ortolani CLF, Kamitsuji IKN, Rodrigues CPF. Normatização e padronização da localização e abreviação dos pontos cefalométricos utilizados nas analises cefalométricas em norma lateral de Ricketts e Shwarz-Faltin. Rev Inst Ciênc Saúde. 2008; 26(3):328-33.

12 - Winter GB. Impacted mandibular third molar. St. Louis: American Medical Book; 1926. 
13 - Little RM. The Irregularity Index: A quantitative score of mandibular anterior alignment. Am J Orthod. 1975; 68(5): 554-564.

14 - Hasegawa Y, Terada K, Kageyama I, Tsuchimochi T, Ishikawa F, Nakahara S. Influence of third molar space on angulation and dental arch crowding. Odontology. 2013; 101(1):22-28.

15 - Ganss C, Hochban W, Kielbassa AM, Umstadt HE. Prognosis of third molar eruption. Oral Surg Oral Med Oral Pathol. 1993; 76(6): 688-693.

16 - Ponnada SR, Ganugapanta VR, Perumalla KK, Nadeeq MA, Harini T, Mandaloju SP. Airway Analysis in Skeletal Class I and Class II Subjects with Different Growth Patterns: A 2D Cephalometric Study. J Pharm Bioallied Sci. 2020; 12(Suppl 1): S161-S167.

17 - Camci H, Salmanpour F. Cephalometric Evaluation of Anterior Cranial Base Slope in Patients with Skeletal Class I Malocclusion with Low or High SNA and SNB Angles. Turk J Orthod. 2020; 33(3): 171-176.

18 - Seifi M, Boroujeni MJ, Tabrizi R, Tahmasbi S. Association between Lateral Cephalometric Changes in X-Y Coordinate System and Profile Changes among Skeletal Class III Patients after Orthognathic Surgery. World J Plast Surg. 2020; 9(3): 282-289.

19 - Chaves-Neto HDM, Carvalho FN, Maciel SM, Olate S, Mazzonetto R. Existe relación entre apiñamiento dentario antero-inferior y terceros molares inferiores?. Acta Odontol Venezoelana. 2010;48(4):online.

20 - Tsiopas N, Nilner M, Bondemark L, Bjerklin K. A 40 years follow-up of dental arch dimensions and incisor irregularity in adults. Eur J Orthod. 2013; 35(2): 230-235.

21 - Lindauer SJ, Laskin DM, Tüfekçi E, Taylor RS, Cushing BJ, Best AM. Orthodontists' and surgeons' opinions on the role of third molars as a cause of dental crowding. Am J Orthod Dentofacial Orthop. 2007; 132(1): 43-48.

22 - Tüfekçi E, Svensk D, Kallunki J, Huggare J, Lindauer SJ, Laskin DM. Opinions of American and Swedish Orthodontists about the Role of Erupting Third Molars as a Cause of Dental Crowding. Angle Orthod.2009; 79(6):1139-1142.

23 - Gavazzi M, Angelis D, Blasi S, Pesce P, Lanteri V. Third Molars and Dental Crowding: Different Opinions of Orthodontists and Oral Surgeons Among Italian Practitioners. Prog Orthod. 2014; 15(1):60. 


\title{
The influence of third molars in the lower incisor crowding
}

\begin{abstract}
Introduction: The time of eruption of the lower third molars can coincide with the crowding of the lower incisors. The third molar as one of the etiological factors of the lower crowding is not yet fully accepted and can lead to unnecessary treatments and uncertain of the orthodontic treatment results. Aim: Correlate the retromolar space and lower incisors crowding. Material and Methods: The facial growth pattern and the participants' retromolar distance were determined by analyzing the cephalometric radiography. Little's irregularity index was calculated, based on the model analysis, to determine the severity of tooth crowding. Panoramic radiographies were used to assess the presence and position of third molars. Results: The mesofacial pattern was the most frequent $(66.7 \%)$, followed by brachyfacial (33.3\%). Most patients had moderate crowding (52.4\%), followed by minimal crowding (28.6\%). There was no statistically significant difference in Little's index considering the facial typology. There was no correlation between retromolar space and Little's index. Conclusion: There is no significant correlation between the retromolar space and dental crowding, suggesting that the presence of the third molar has no influence on the anteroinferior crowding.
\end{abstract}

KEYWORDS: Third molar; Malocclusion; Orthodontics.

\section{Como citar este artigo}

Teixeira KVS, Souza MEA, Castro TGR, Martins AFL. A influência dos terceiros molares no apinhamento de incisivos inferiores. Rev Odontol Bras Central 2021;30(89):357-371. DOI:10.36065/ robrac.v30i89.1514 